\title{
Scatter free imaging for the improvement of breast cancer detection in mammography
}

\author{
F.H.Green ${ }^{1}$, M.C.Veale ${ }^{2}$, M.D.Wilson ${ }^{2}$, P.Seller ${ }^{2}$, J.Scuffham ${ }^{3}$, \\ S.Pani ${ }^{1}$ \\ ${ }^{1}$ University of Surrey, Guildford, GU2 7XH, UK \\ ${ }^{2}$ STFC Rutherford Appleton Laboratories, Didcot, UK \\ ${ }^{3}$ Department of Nuclear Medicine, Royal Surrey County Hospital, Guildford, UK \\ E-mail: faith_hannah_green@yahoo.co.uk
}

23 September 2016

\begin{abstract}
In mammography, the reduction of scattered X-rays is vital due to the low contrast or small dimension of the details that are searched for. The typical method of doing so in current conventional mammography is the anti-scatter grid. The disadvantage of this method is the absorption of a proportion of the primary beam and therefore an increase in dose is required to compensate for the loss of counts.

An alternative method is proposed, using quasi-monochromatic beams and a pixellated spectroscopic detector. As Compton-scattered X-rays lose energy in the scattering process, they are detected at a lower energy in the spectrum. Therefore the spectrum can be windowed around the monochromatic energy peak, removing the scattered Xrays from the image.

The work presented here shows contrast improvement of up to $50 \%$ and contrast to noise ratio improvements of around $20 \%$ for scatter free imaging in comparison to full spectrum imaging. Contrast improvements of around $45 \%$ were found when comparing scatter free images to conventional polychromatic imaging for both the low contrast test object and the Rachel anthropomorphic breast phantom.
\end{abstract}

Keywords: Mammography, hyperspectral imaging, contrast 


\section{Introduction}

In mammography, details that could suggest the presence of cancer include masses and calcifications that feature low contrast or small size, respectively, making them difficult to detect. To improve the chance of correctly identifying these features, dedicated imaging systems are used [Bushberg et al., 2011].

Breast tissue is very sensitive to radiation so the dose must be kept at a minimum [Ronckers et al., 2005]. As lower dose leads to worse image quality, there is a trade off between the two that needs to be considered.

One of the main causes of image degradation in X-ray imaging is Compton scattering [Flower, 2012]. The current method to reduce the amount of Compton-scattered X-rays is the anti-scatter grid. This is a series of lead septa that absorb X-rays not travelling parallel to the primary beam. The disadvantage of this method is that it also absorbs a proportion of the primary beam, and as a result an increase in the dose is required to compensate [Sprawls, 1995].

There are other alternative methods for scatter removal for the use of mammography. The slot scanning method uses collimators to produce a fan beam to scan across the breast. The beam is then collimated again after interaction with the breast in order to stop scattered X-rays. The narrow shape of the beam reduces the amount of scattered photons, therefore improving image quality [Besson et al., 2002]. However, this method does allow for a proportion of scatter to reach the image which leads to less of a reduction in dose than with a completely scatter free imaging system [Oppelt, 2011].

Some post processing approaches involve modelling of scatter produced depending on breast thickness and density [Darboux and Dinten, 1997]. This technique is extremely complex as the scatter produced from breast tissue is highly dependent on the specific density which varies in every woman imaged [Wang et al., 2015] [Ducote and Molloi, 2010].

This project investigates a way of removing scattered X-rays without requiring an increase in the dose. When X-rays scatter due to Compton scattering they lose a proportion of their energy in the process, and will therefore have a lower energy than the primary beam in the detected spectrum. A pixellated spectroscopic detector produces both spatial and spectroscopic information. Combining the detector with a monochromatic X-ray source means the Compton-scattered component of the beam can be windowed out of the spectrum, hence producing an essentially scatter free image.

The concept of monochromatic imaging has been investigated previously. When a polychromatic beam is incident on a perfect crystal with a lattice spacing of $d$ and at an angle $\theta$, only integer $(n)$ multiples of the wavelength $(\lambda)$ will constructively interfere and diffract from the crystal. This relationship is described by Bragg's law, equation 1.1 [Ahmed, 2014].

$$
n \lambda=2 d \sin \theta
$$

Monochromatic imaging using a crystal has two main benefits. Firstly it removes the lower and higher energy X-rays from the beam. Low energy X-rays are absorbed, 
increasing the dose without contributing to the image. High energy X-rays give rise to low contrast and are more likely to scatter decreasing the image quality. The second benefit is the tuneability of the beam by simply changing the angle of the crystal. This leads to the optimum energy being used depending on the thickness and density of the breast being imaged.

Typically, studies involving monochromatic X-rays have been carried out at synchrotron sources, where the high intensity of the white beam allows sufficient intensity after monochromatisation [Burattini et al., 1995] [Moeckli et al., 2000]. This work has had clinical trials which have shown an improvement in contrast and small detail detection when compared to conventional mammographic imaging [Castelli et al., 2011][Longo et al., 2014]. However, this approach is expensive and impractical for screening due to the poor accessibility of a synchrotron [Baldelli et al., 2003].

A conventional X-ray source is not intense enough to produce a sufficiently intense monochromatic beam for imaging. To solve this problem, a mosaic crystal may be used. This differs from a perfect crystal as it has a range of lattice orientations that will produce a small spread in the energies diffracted and therefore increase the overall intensity of the beam [Freund et al., 1996], as demonstrated in figure 1.

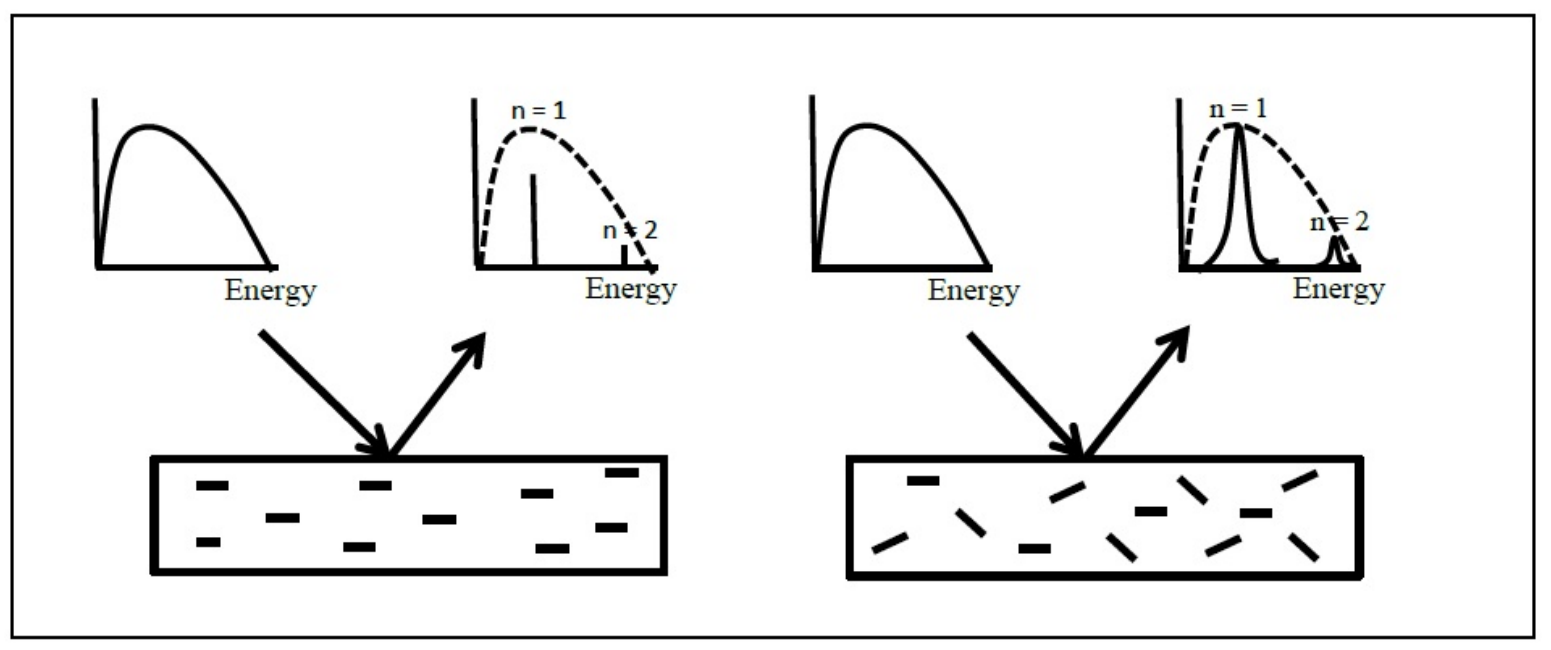

Figure 1. The effects of a mosaic crystal (right) on the intensity and resolution of the diffracted energy peaks in comparison to a perfect crystal (left).

Work using a conventional X-ray source combined with a mosaic crystal to produce a monochromatic beam has been done by the research group in Ferrara, Italy. A prototype was developed that, using several crystals and a scanning motion of the beam, could produce a monochromatic beam with an intensity and beam dimension large enough for clinical imaging [Gambaccini et al., 1999]. The study showed that the prototype could image with a comparable image quality to conventional mammography but with half the dose [Baldelli et al., 2005].

A pixellated spectroscopic detector measures both spatial and spectral information. 
There has been work on imaging with a pixellated spectroscopic detector by the Medipix detector group. Medipix2 has two pulse height discriminators, that can be set so that two images are simultaneously acquired; one with an energy below the first discriminator and one with and energy above the second discriminator. By combining these images, an image of the energy band between the two thresholds is formed [Melzer et al., 2008]. This method has the benefit of only detecting in the energy range required. This technique has benefits in many fields including the aerospace industry where detecting low energy of X-rays is important [Pinsky et al., 2014]. Further development is Medipix3 that has up to 8 thresholds that can be assigned which further increases the spectroscopic capabilities of the system [Ballabriga et al., 2011].

Other photon counting systems have been developed or are in the development stage that have possible advantages for improving image quality. For mammography these are Microdose by Philips and XCounter by XCounter, Stockholm. Microdose, that using the slot scanning method described earlier, is a commercially available product [Lundqvist et al., 2003]. Comparisons between Microdose and conventional detectors found that Microdose outperforms flat panel detectors in terms of dose and noise [Monnin et al., 2007]. XCounter is based on avalanche detector technology. This method has an excellent signal to noise ratio [Thunberg et al., 2002].

Photon counting detectors have been combined with Computed Tomography (CT) to produce spectral CT. Breast CT has the benefits of being able to distinguish overlapping features in dense breast tissue better than in conventional mammography [Boone et al., 2001]. Spectral CT combines the positional information of conventional CT with spectral information. This allows for imaging optimisation by selecting the optimal energy range depending on the details that are being detected [Brahme, 2014]. Results have shown an improvement in the CNR for spectral CT over conventional CT imaging [Shikhaliev and Fritz, 2011]. There is, however, still the issue of cost for such a system to be used in screening.

The HEXITEC detector used in this study has the added benefit of being able to obtain the entire detected spectrum instead of selecting energy bands. This produces an energy resolution of $0.8 \mathrm{keV}$ at $59.9 \mathrm{keV}$. The work in the literature so far has either used monochromatic X-rays or spectroscopic imaging. The very high energy resolution of HEXITEC means that the two methods can be combined as the monochromatic peak can be resolved from scattered photons in the detected spectrum. Therefore, scatter can be windowed from the spectra around the mononchromatic peak, producing scatter-free images. This work demonstrates the improvement in contrast before and after windowing of the monochromatic X-ray spectrum.

Conventional mammography uses a polychromatic X-ray beam. For comparisons to be made between scatter-free imaging and the current mammographic technique, polychromatic images were acquired and the image quality compared. As an additional study, an anthropomorphic breast phantom was imaged with the monochromatic and polychromatic beams in order to test the image quality improvements with a test object more realistic to breast tissue. 


\section{Materials and Methods}

\subsection{Experimental Setup}

The experimental setup, shown in figure 2, consists of a Hamamatsu L6732-01 X-ray source with a tungsten anode operated at voltages up to $60 \mathrm{kVp}$, a $10 \mathrm{~mm}$ thick brass collimator with a $1 \mathrm{~mm}$ x $20 \mathrm{~mm}$ slit, a Highly Ordered Pyrolytic Graphite crystal combined with a goniometer, a test object and a pixellated spectroscopic detector.

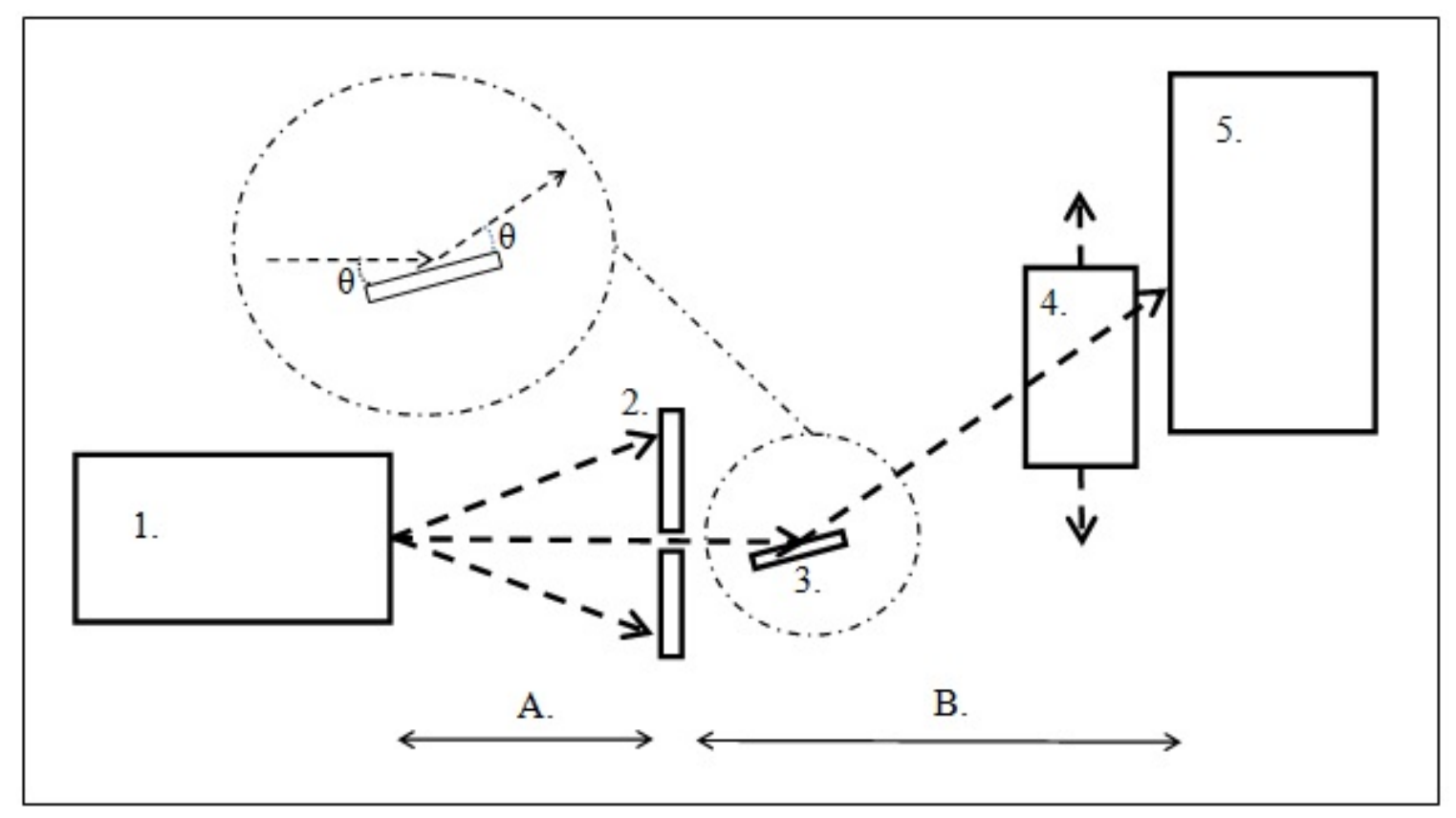

Figure 2. The monochromator setup. On the diagram, 1 is the $\mathrm{X}$-ray source, 2 is the collimator, 3 is the mosaic crystal, 4 is the test object and 5 is the HEXITEC spectroscopic detector. The angle $\theta$ is shown. A is the source-to-collimator distance and is equal to $60 \mathrm{~cm}$. B is the collimator-to-detector distance and is equal to $41 \mathrm{~cm}$.

Monochromator The monochromator consists of a $2 \mathrm{~cm} \times 2 \mathrm{~cm} \times 0.2 \mathrm{~cm}$ Highly Ordered Graphite (HOPG) crystal mounted on a goniometer for angle adjustment. The crystal has a mosaic spread of $(0.4 \pm 0.1)^{\circ}$ [Optigraph, 2011].

HEXITEC The HEXITEC system consists of a $2 \mathrm{~cm} \times 2 \mathrm{~cm} \times 0.1 \mathrm{~cm}$ CdTe wafer, bump-bonded to an $80 \times 80$ readout array, each pixel producing an energy spectrum of the detected X-rays. The detector array has a pixel pitch of $250 \mu \mathrm{m}$, a inter pixel spacing of $50 \mu \mathrm{m}$ and an energy resolution of $0.8 \mathrm{keV}$ at $59.5 \mathrm{keV}$ [Veale et al., 2012]. Each pixel has an identical integrated circuit consisting of amplifiers and charge shapers. The energy of each photon detected by the detector is calculated using a peak hold circuit that measures the magnitude of the pulse produced by each photon that has interacted with the CdTe [Wilson et al., 2013]. The detector is cooled at a sub-room temperature 
in order to improve the spectral resolution. For this study it was cooled at $10{ }^{\circ} \mathrm{C}$ which produced a sensor temperature of approximately $19{ }^{\circ} \mathrm{C}$.

Low contrast test object In order to investigate the image quality of the scatter free images a low contrast test object was used, schematically shown in figure 3 . This consisted of a $10 \mathrm{~cm}$ x $10 \mathrm{~cm}$ x $0.5 \mathrm{~cm}$ Perspex block with three, $5 \mathrm{~mm}$ diameter holes. One hole was filled with wax, one with nylon and the final hole was left empty. The test object could then be combined with different amounts of Perspex to vary the thickness of the overall test object.

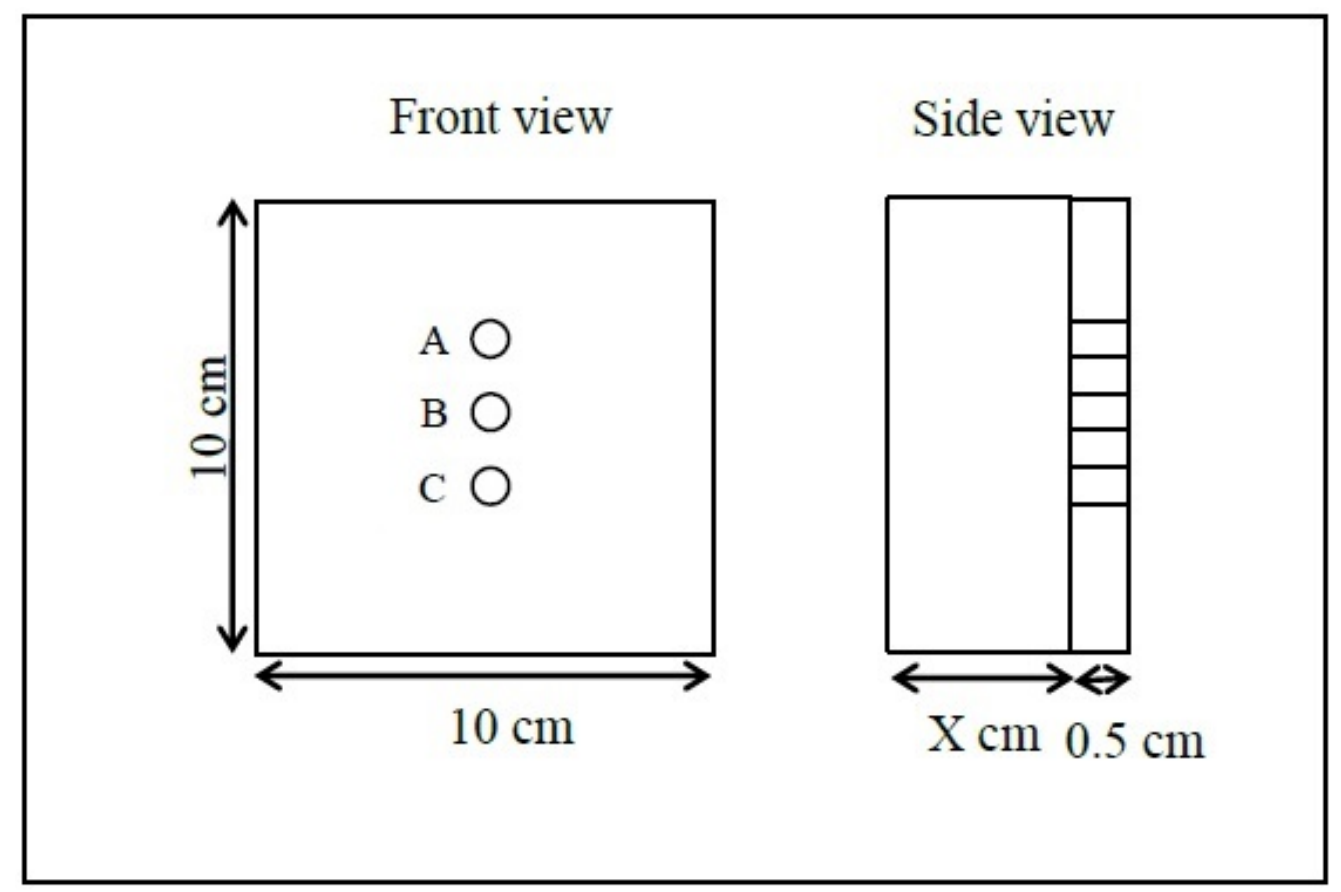

Figure 3. a schematic of the low contrast test object from a front and side view. $\mathrm{X}$ $\mathrm{cm}$ represents the added perspex thickness that can be varied to change the thickness of the test object. A, B and C represent the Nylon, Wax and Air filled sets of holes.

Rachel anthropomorphic phantom For a realistic impression of how the scatter removal would affect clinical imaging, the Rachel anthropomorphic breast phantom was imaged. The phantom is made from three components: a breast-equivalent material known as BR12 for the main breast region [Ducote and Molloi, 2010], a high resolution film that inputs the finer details and the surrounding acrylic case.

\subsection{Contrast and contrast to noise ratio (CNR)}

Contrast is the ability to distinguish between details and the surrounding background region [Bushberg et al., 2011]. The contrast is defined using equation 2.1, where $M_{\text {in }}$ 
and $M_{\text {out }}$ and the average signals inside and outside a detail [Bushberg et al., 2011].

$$
C=\frac{\left|M_{\text {out }}-M_{\text {in }}\right|}{M A X\left(M_{\text {out }}, M_{\text {in }}\right)}
$$

An alternative measure of the image quality that also takes into account noise is the contrast to noise ratio (CNR) given by equation 2.2 [Bushberg et al., 2011]. Here $\sigma_{\text {out }}$ is the standard deviation in the background signal.

$$
C N R=\frac{\left|M_{\text {out }}-M_{\text {in }}\right|}{\sigma_{\text {out }}}
$$

\subsection{Mean Glandular Dose (MGD)}

Mammography is used for screening and diagnostic imaging. When used for screening the dose is imparted into potentially healthy breast tissue and therefore it needs to be closely monitored to prevent damage to healthy tissue.

The formation of cancer (carcinogenesis) occurs in glandular breast tissue and therefore it is the dose within this tissue type that needs to be monitored. To do this the Mean Glandular Dose (MGD) is calculated using equation 2.3 where $\chi$ is the entrance skin air kerma and $g$ is the air kerma to MGD conversion factor.

$$
M G D=\chi g
$$

The entrance skin air kerma is a measure of the dose in the air rather than the tissue of the body and is used as it is much easier to calculate. It is calculated using equation 2.4, where $N_{0}$ is the number of incident photons per unit area, $(\mu / \rho)_{\text {air }}$ is the mass attenuation coefficient of air and $\mathrm{E}$ is the X-ray energy. Note that the IQF in the next section is independent of $N_{0}$ and therefore this value was not calculated in this study. For the study in [Green et al., 2015] an $N_{0}$ value of 20,000 was chosen.

$$
\chi=N_{0}\left(\frac{\mu}{\rho}\right)_{a i r} E
$$

The component $g$ converts this dose into the mean glandular dose. It is determined using computer simulations that takes into account the quality of the beam, the breast thickness and the half value layer (HVL) [Dance et al., 2000]. The HVL is the thickness of aluminium needed to reduce the dose by half and is calculated using equation 2.5 where $\mu$ is the attenuation coefficient of aluminium. As the attenuation coefficient is energy dependent, for a polychromatic beam the HVL is calculated using several thicknesses of aluminium and doing an exponential fit. This study focuses on monochromatic energies and therefore the HVL is calculated using equation 2.5 where a single value of the attenuation coefficient is inserted for the energy concerned.

$$
H V L=\frac{0.693}{\mu_{\mathrm{Al}}}
$$




\subsection{Image Quality Factor}

The optimum energy required for imaging in mammography is dependent on the thickness of the breast being imaged because a thicker breast requires higher energy to penetrate the tissue. Higher energies result in lower attenuation and therefore lower dose, for a certain level of statistics on the detector, but the lower attenuation also reduces the contrast of the image. Lower energies result in an increase in the dose as they are absorbed by the tissue. Therefore, a trade off is required between the dose to the patient and the image quality.

In order to identify the ideal monochromatic energy to image differing thicknesses of test object the image quality factor was calculated. This takes into account the dose and the image quality, equation 2.6 where CNR is the contrast to noise ratio (equation 2.2) and MGD is the mean glandular dose (equation 2.3) [Aichinger et al., 2011]. The CNR was used instead of SNR as the size of detail being imaged was kept constant.

$$
I Q F=\frac{C N R^{2}}{M G D}
$$

The calculations of the IQF are explained in previous work [Green et al., 2015]. Table 1 lists the calculated optimum energies for a range of thicknesses and this was used for all the imaging in this study.

\begin{tabular}{|c|c|}
\hline Perspex thickness $(\mathrm{mm})$ & Optimum energy $(\mathrm{keV})$ \\
\hline 30 & 17 \\
\hline 40 & 18 \\
\hline 50 & 19 \\
\hline 60 & 20 \\
\hline 70 & 21 \\
\hline
\end{tabular}

Table 1. The optimum monochromatic energies for a range of object thicknesses. The values were calculated for both the Nylon and wax details of the low contrast test object and averaged to find these optimum energy values [Green et al., 2015].

\subsection{Image Acquisition and reconstruction}

The beam produced by the described setup has dimensions of $2 \mathrm{~cm} \times h \mathrm{~cm}$, where $h$ is equal to the height of the beam and is dependent on the angle between the beam and the crystal. A more grazing angle produces a beam with a smaller height and a less grazing angle produces a beam with a bigger height [Green et al., 2014].

In order to image an object that is larger than the beam height, multiple images were needed. This involved mounting the object on a linear motor that moved the object by the height of the beam in between acquisitions. These multiple images were then combined together using a MATLAB program to produce the final image. Flat-field 
corrections were applied to remove any detector related artifacts and to correct for nonuniformities of the beam. Then the spectra could be windowed around the user selected region, an example monochromatic spectrum is shown in figure 4.

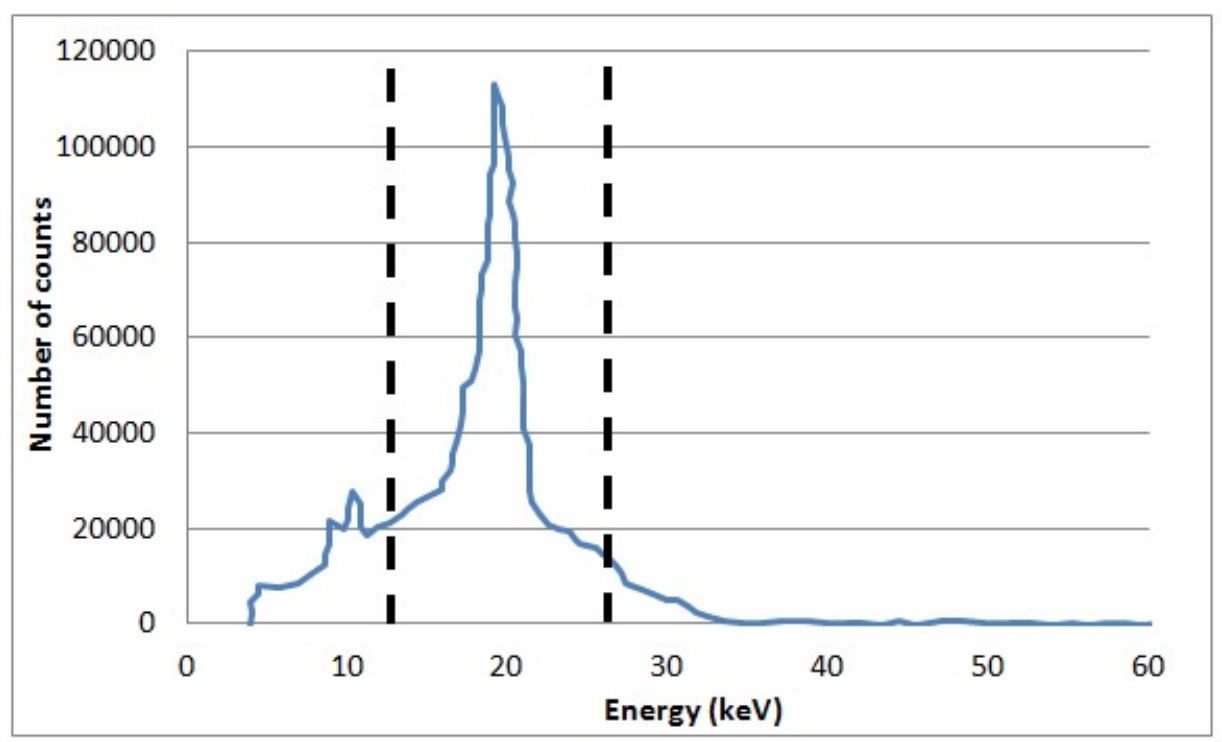

Figure 4. An example experimental monochromatic spectrum. The black lines represent the lower and higher regions that the spectrum is windowed around to remove scatter.

\section{Results}

Preliminary imaging Imaging using a monochromatic beam results in a lower beam intensity which can result in a poor image quality. In order to increase the intensity of the monochromatic beam a higher tube voltage could be used. Higher tube voltages will result in higher order peaks forming in the spectrum. These can be windowed out but it is possible that scatter from these higher order peaks could overlap the lower monochromatic peak and decrease image quality. To investigate this effect, images of the $4 \mathrm{~cm}$ thick low contrast phantom were taken with a monochromatic energy of 18 $\mathrm{keV}$. Tube voltages of $35 \mathrm{kVp}$ and $50 \mathrm{kVp}$ were chosen in order to image with and without the presence of the second order peak $(36 \mathrm{keV})$.

To analyse the resulting images the contrast (equation 2.1) was calculated for the two different low contrast details (wax and nylon), shown in figure 2 .

The contrast values show that there is little difference between the lower and higher tube voltages in terms of image quality. Therefore, scatter from the higher order peak is not interfering with the first order peak and so images can be acquired with higher tube voltages.

Low contrast imaging The test object was imaged for a $4 \mathrm{~cm}, 5 \mathrm{~cm}$ and $6 \mathrm{~cm}$ thickness of Perspex using the optimal imaging parameters determined using the IQF. A tube 
Table 2. The contrast values for the wax and Nylon details are shown for both the $35 \mathrm{kVp}$ and $50 \mathrm{kVp}$ tube voltages.

\begin{tabular}{lll}
\hline Detail & $35 \mathrm{kVp}$ contrast & $50 \mathrm{kVp}$ contrast \\
\hline Wax & $0.15 \pm 0.03$ & $0.12 \pm 0.04$ \\
Nylon & $0.06 \pm 0.04$ & $0.10 \pm 0.05$ \\
\hline
\end{tabular}

voltage of $60 \mathrm{kVp}$ was chosen in order to provide a sufficient beam intensity for imaging. The images were then formed using the methods described in previous work [Green et al., 2015]. Images were formed using both the full energy spectrum and the energy spectrum windowed around the monochromatic peak, figure 5. Figure 6 shows the full spectrum and scatter free profiles for the $6 \mathrm{~cm}$ thickness data. For each object thickness and each of the lowest contrast details (Wax and Nylon), the contrast and the CNR was calculated and shown in tables 3 and 4 .

$4 \mathrm{~cm}$

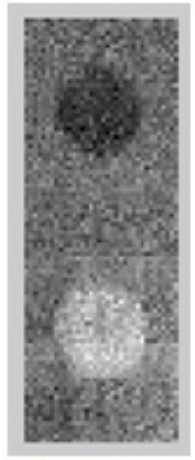

Before

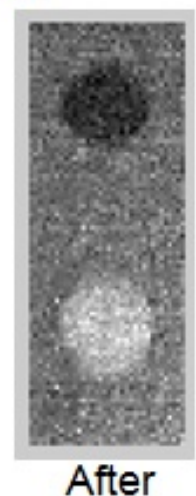

$5 \mathrm{~cm}$

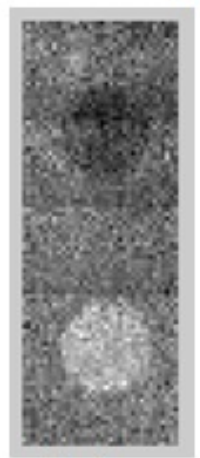

Before

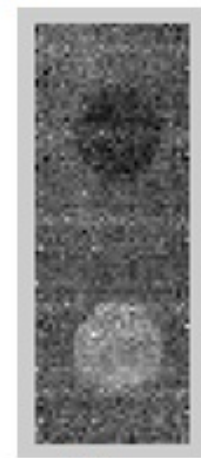

After
$6 \mathrm{~cm}$

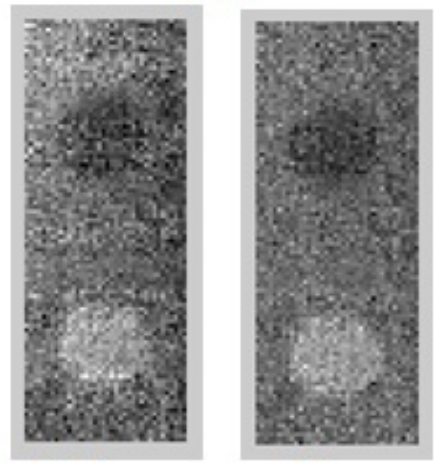

Before
After

Figure 5. The reconstructed images for 4,5 and $6 \mathrm{~cm}$ thickness low contrast test object; shown with both the full spectrum and windowed around the monochromatic energy peak.

Table 3. The contrast values for the wax and nylon details for 4,5 and $6 \mathrm{~cm}$ of test object. Values both before and after the spectrum was windowed around the monochromatic peak are shown.

\begin{tabular}{llll}
\hline Object thickness & Detail & Full spectrum contrast & Scatter-free contrast \\
\hline $40 \mathrm{~mm}$ & Wax & $0.045 \pm 0.002$ & $0.090 \pm 0.004$ \\
$40 \mathrm{~mm}$ & Nylon & $0.048 \pm 0.002$ & $0.099 \pm 0.005$ \\
$50 \mathrm{~mm}$ & Wax & $0.031 \pm 0.001$ & $0.062 \pm 0.003$ \\
$50 \mathrm{~mm}$ & Nylon & $0.040 \pm 0.002$ & $0.082 \pm 0.004$ \\
$60 \mathrm{~mm}$ & Wax & $0.024 \pm 0.001$ & $0.048 \pm 0.002$ \\
$60 \mathrm{~mm}$ & Nylon & $0.031 \pm 0.002$ & $0.062 \pm 0.003$ \\
\hline
\end{tabular}




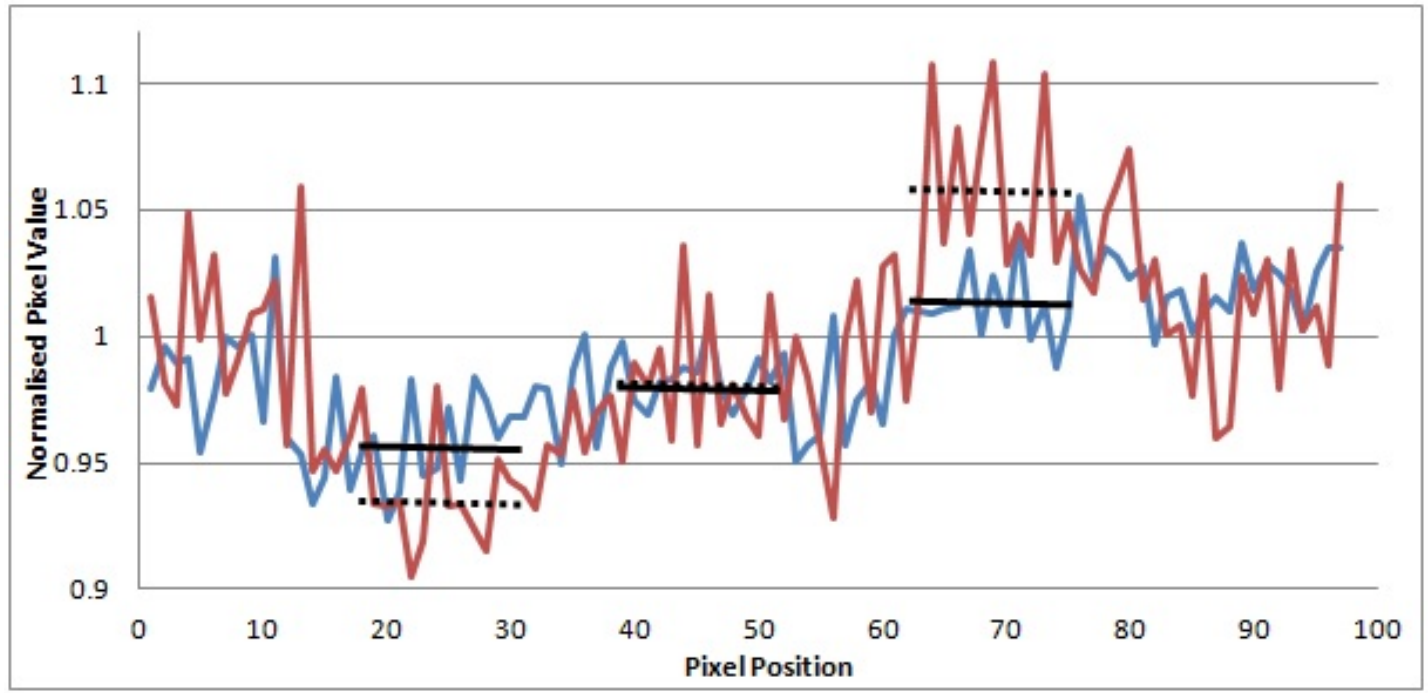

Figure 6. The full spectrum (blue) and scatter free (red) profiles for the $6 \mathrm{~cm}$ thickness of perspex. The full (full spectrum) and dotted (scatter free) lines represent the regions that the contrast was calculated from. The lower values to the left represent the wax detail, the higher points to the right represent the Nylon detail and the points in the middle represent the background values. The profiles were normalised to the average value in the background region.

Table 4. The CNR values for the wax and nylon details for 4,5 and $6 \mathrm{~cm}$ of test object. Values both before and after the spectrum was windowed around the monochromatic peak are shown.

\begin{tabular}{llll}
\hline Object thickness & Detail & Full spectrum CNR & Scatter-free CNR \\
\hline $40 \mathrm{~mm}$ & Wax & $259 \pm 9$ & $379 \pm 8$ \\
$40 \mathrm{~mm}$ & Nylon & $378 \pm 11$ & $481 \pm 12$ \\
$50 \mathrm{~mm}$ & Wax & $258 \pm 8$ & $324 \pm 10$ \\
$50 \mathrm{~mm}$ & Nylon & $316 \pm 10$ & $401 \pm 11$ \\
$60 \mathrm{~mm}$ & Wax & $175 \pm 8$ & $219 \pm 9$ \\
$60 \mathrm{~mm}$ & Nylon & $234 \pm 11$ & $301 \pm 15$ \\
\hline
\end{tabular}

From the images in figure 5 there is a visible difference between the full spectrum and scatter free images, especially with the $6 \mathrm{~cm}$ object thickness where the details of the scatter free image are much clearer. This is confirmed with the profile plot in figure 6 . The points used to calculate the scatter free contrast are much more deviated from the background than the full spectrum image showing a greater contrast for the scatter free data.

The measurement of contrast, table 3, shows a $50 \%$ increase when the scatter was removed from the image. Windowing around the monochromatic peak does result in an increase noise due to a decrease in counts. In order to show that there is an increase in detail visibility even with this increase in standard deviation the contrast to noise ratio was calculated. These values, shown in table 4 , show a $20 \%$ increase in image quality 
for the scatter free images.

These results show that there is a significant improvement in the image quality of low contrast details once the scatter has been removed from the image by windowing the energy spectrum.

As a final comparison the low contrast test object was imaged with a full polychromatic beam with an average energy equal to the monochromatic energies used to image each thickness. To produce average energies of 18, 19 and $21 \mathrm{keV}$ using a tungsten anode $\mathrm{X}$-ray tube, tube voltages of 35,38 and $45 \mathrm{kVp}$ were used respectively. These were calculated by modelling spectra using the spectrum generator SpekCalc [Evans, 2007] [Poludniowski, 2007] [Poludniowski et al., 2009]

Figure 7 shows the full polychromatic images of the test object and table 5 shows the contrast values for each detail and object thickness. Comparing the contrast values of the two images shows that there is about a $45 \%$ increase in contrast for the monochromatic images. However, when comparing the CNR values they are higher for the full polychromatic spectrum. This is due to the significantly higher statistics and therefore lower noise for the polychromatic spectrum. However, the MGD is also significantly higher. To remove the dependence on dose the IQF (equation 2.6) was calculated. Table 6 compares the IQF for the full spectrum and scatter-free images. It shows an increase in IQF of around $65 \%$ for the monochromatic, scatter free images.
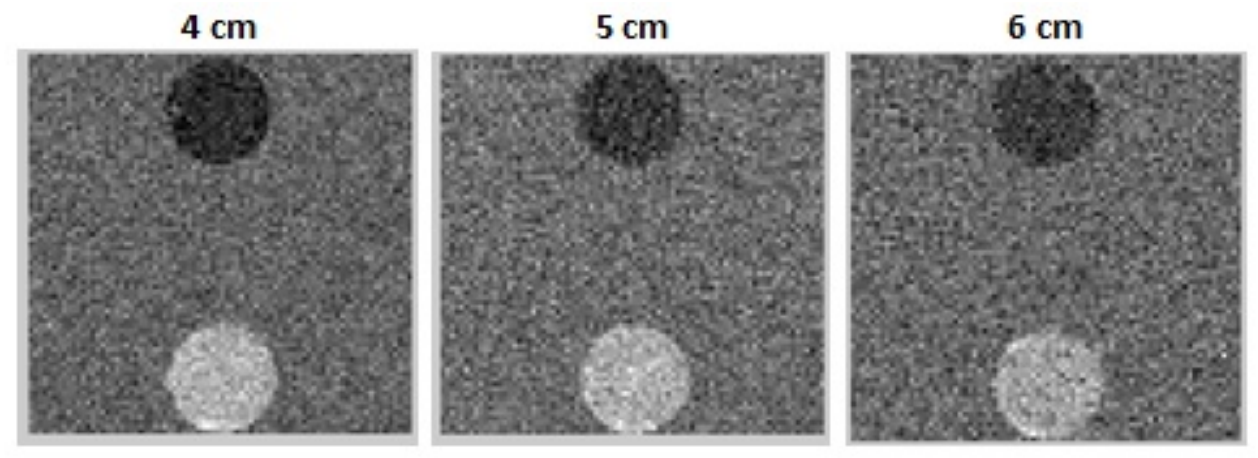

Figure 7. the full spectrum images for 4,5 and $6 \mathrm{~cm}$ thickness low contrast test object.

Rachel anthropomorphic phantom The Rachel anthropomorphic breast phantom was imaged with a monochromatic energy of $21 \mathrm{keV}$, chosen from the IQF measurements. Two regions were imaged and the spectra windowed to produce full spectrum and scatter free images; shown in figure 8.

The images show some enhancement between the fatty and glandular regions. In order to quantify this enhancement in the image quality some profiles across the images were taken and compared for before and after the scatter was removed from the image. Two profiles were taken from each image, one of a single row and the other a summed profile of all the rows, the location of these profiles are shown by the dotted line on the images 
Table 5. the contrast and CNR values for the wax and nylon details and different test object thickness imaged with a conventional full spectrum. Tube voltages of 35, 38 and $45 \mathrm{kVp}$ were used for the 4,5 and $6 \mathrm{~cm}$ test object thicknesses respectively.

\begin{tabular}{llll}
\hline Object thickness & Detail & Full polychromatic contrast & full polychromatic CNR \\
\hline $4 \mathrm{~cm}$ & Wax & $0.048 \pm 0.002$ & $382 \pm 8$ \\
$4 \mathrm{~cm}$ & Nylon & $0.063 \pm 0.003$ & $537 \pm 12$ \\
$5 \mathrm{~cm}$ & Wax & $0.037 \pm 0.002$ & $257 \pm 9$ \\
$5 \mathrm{~cm}$ & Nylon & $0.049 \pm 0.002$ & $361 \pm 10$ \\
$6 \mathrm{~cm}$ & Wax & $0.029 \pm 0.001$ & $246 \pm 8$ \\
$6 \mathrm{~cm}$ & Nylon & $0.034 \pm 0.002$ & $300 \pm 11$ \\
\hline
\end{tabular}

Table 6. the IQF values for the wax and nylon details and different test object thickness imaged with a conventional full spectrum and a monochromatic spectrum with scatter removed.

\begin{tabular}{llll}
\hline Object thickness & Detail & Full polychromatic IQF & Scatter-free IQF \\
\hline $4 \mathrm{~cm}$ & Wax & $20 \pm 1$ & $64 \pm 5$ \\
$4 \mathrm{~cm}$ & Nylon & $41 \pm 3$ & $150 \pm 10$ \\
$5 \mathrm{~cm}$ & Wax & $21 \pm 2$ & $52 \pm 4$ \\
$5 \mathrm{~cm}$ & Nylon & $42 \pm 3$ & $123 \pm 9$ \\
$6 \mathrm{~cm}$ & Wax & $16 \pm 1$ & $58 \pm 4$ \\
$6 \mathrm{~cm}$ & Nylon & $33 \pm 2$ & $140 \pm 10$ \\
\hline
\end{tabular}

in figure 8. Figure 9 shows the profiles for region 1 of the phantom and figure 10 shows the profiles for region 2 .

Region 1

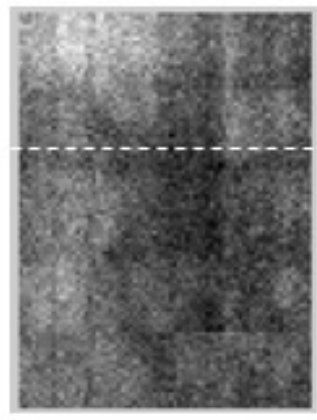

Full spectrum

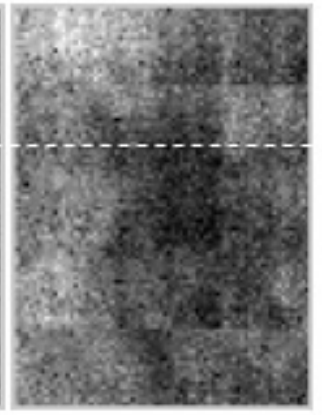

Scatter free
Region 2

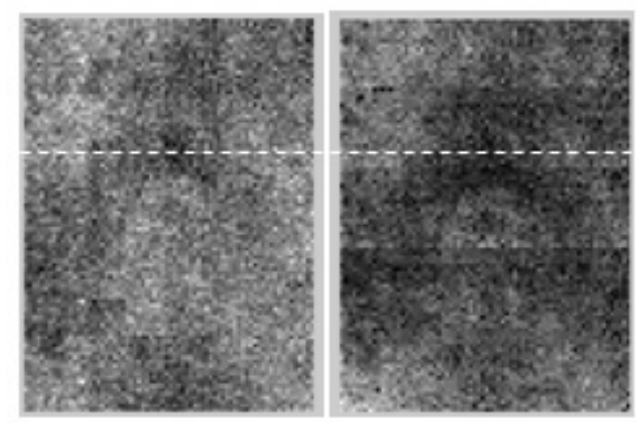

Full spectrum Scatter free

Figure 8. The full monochromatic spectrum and scatter free images of region 1 and region 2 of the breast phantom.

The data shows that when the scatter is removed from the image the fluctuation in the pixel values is more significant. This is as expected due to the lower statistics in the windowed spectrum. The average row data show more clearly that although the pixel 


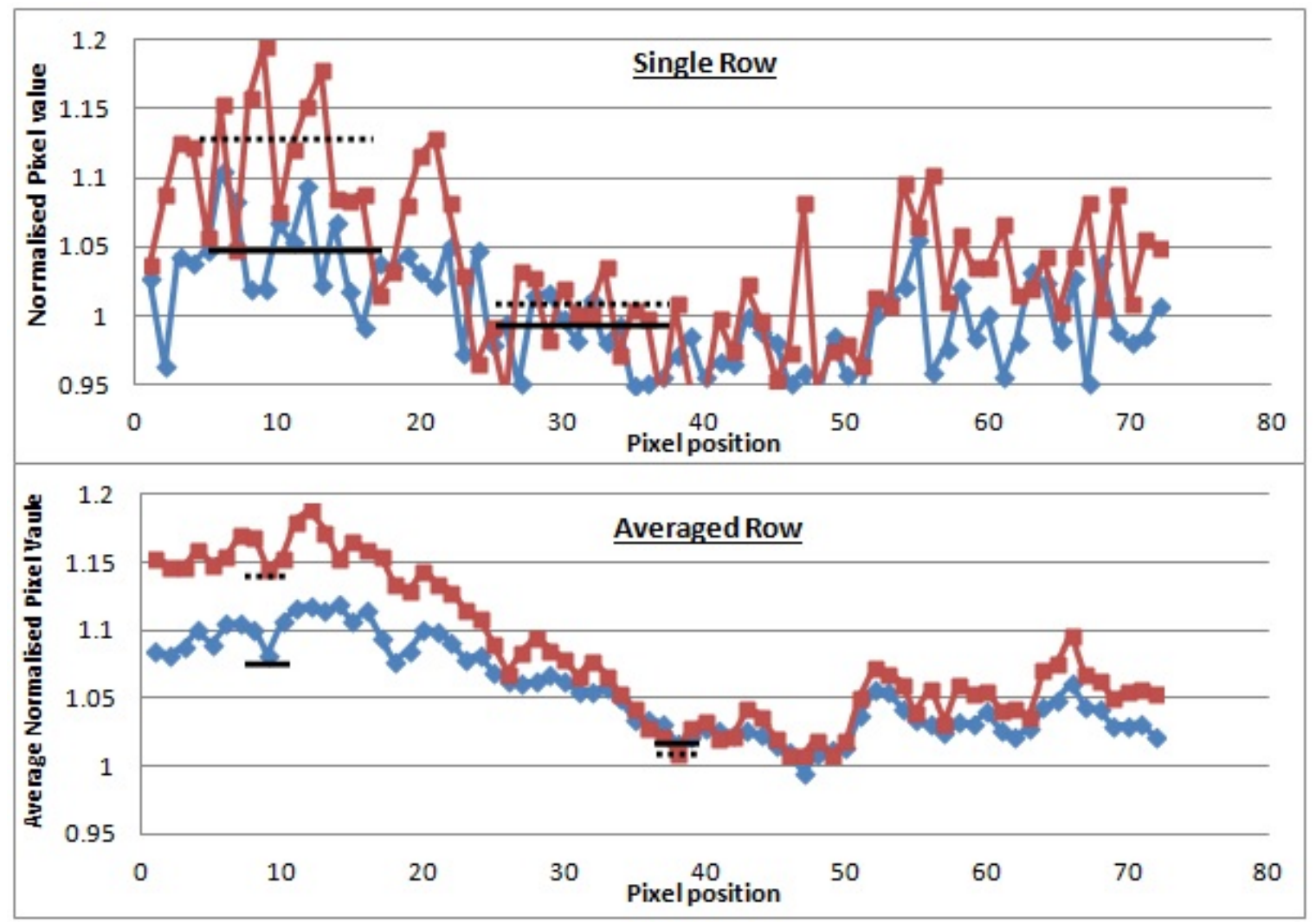

Figure 9. The single row profile (top) and the average row profile (bottom) plots of region 1 of the breast phantom. Scatter-free data (red) and full monochromatic spectrum data (blue) is shown. The pixel values are normalised to the mean pixel value.

values seem to be more variable there is an actual difference in average signal resulting in a greater contrast. To quantify this change in contrast, some regions from each image were identified (shown by the dotted and solid lines on the plots in figure 9 and 10) and the contrast calculated from these points. Table 7 shows that there is an increase in contrast of around $40 \%$ for the individual row and an overall increase in contrast of around $50 \%$ for the averaged data. The averaged data produced a contrast increase similar to that of the $60 \mathrm{kVp}$ low contrast test object data in the previous section. The contrast values of the averaged data are more accurate than the single row data as it was much more difficult to identify features to measure the contrast from on the single row data due to the variation in the data points.

As a final comparison, the monochromatic scatter-free images were compared to the full spectrum images. Figure 11 shows the images of the Rachel phantom for region 1 and region 2 imaged with a full $45 \mathrm{kVp}$ spectrum. This energy was chosen as it produced an average energy equal to the $21 \mathrm{keV}$ monochromatic energy used to produce the scatter free image. The images are smaller than the monochromatic images as a single image was acquired with the same size as the detector, whereas the monochromatic images were obtained by combining several strips. The dotted lines on the full spectrum image 


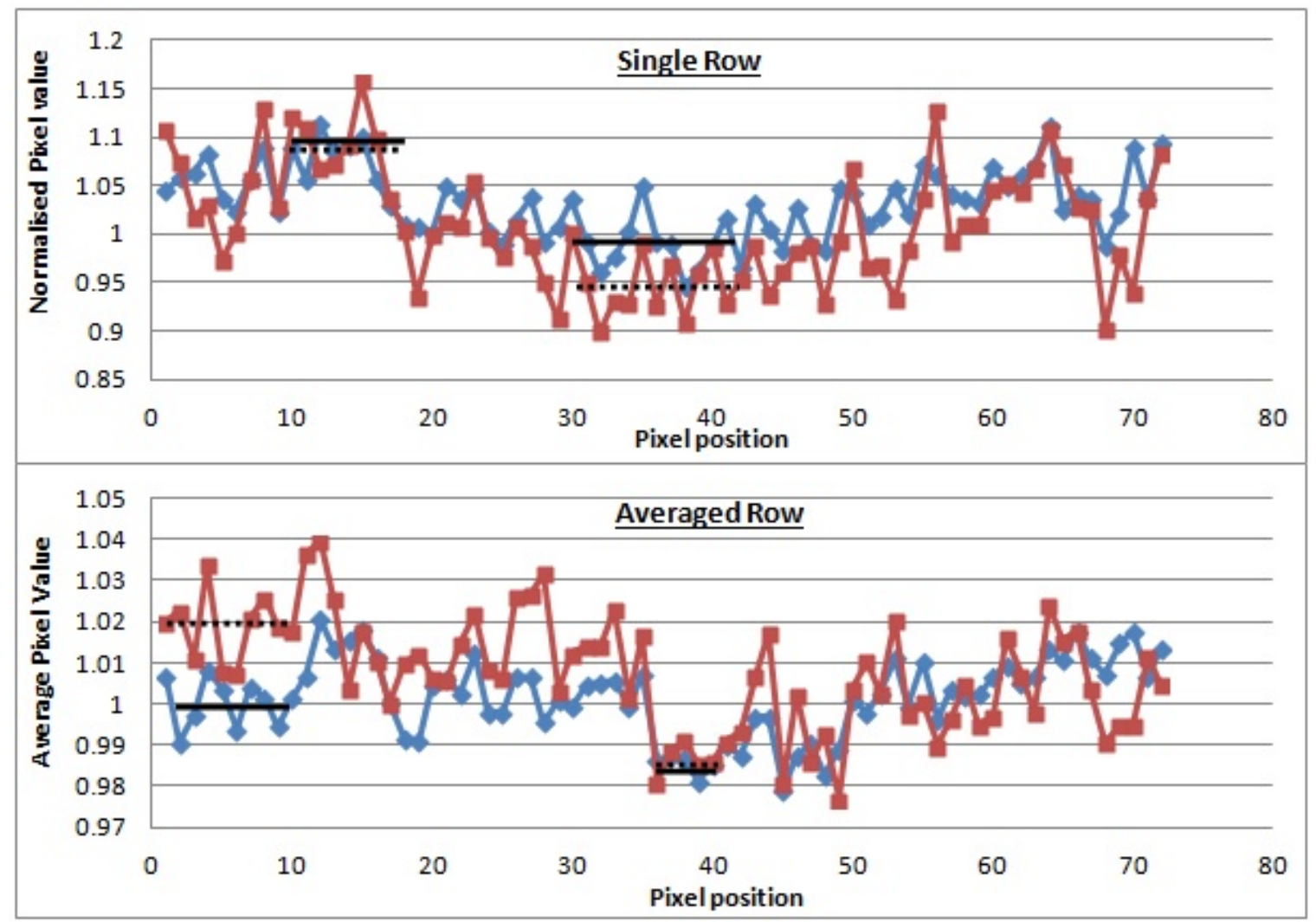

Figure 10. The single row profile (top) and the averaged row profile (bottom) plots of region 2 of the breast phantom. Scatter-free data (red) and full monochromatic spectrum data (blue) is shown. The pixel values are normalised to the mean pixel value.

Table 7. contrast values, with and without scatter for the single row plots and the averaged row plots.

\begin{tabular}{lll}
\hline & Full monochromatic contrast & Scatter-free contrast \\
\hline Region 1 single row & $0.08 \pm 0.01$ & $0.13 \pm 0.01$ \\
Region 2 single row & $0.09 \pm 0.01$ & $0.12 \pm 0.01$ \\
Region 1 averaged row & $0.05 \pm 0.01$ & $0.10 \pm 0.01$ \\
Region 2 averaged row & $0.02 \pm 0.01$ & $0.04 \pm 0.01$ \\
\hline
\end{tabular}

indicate the same row as the dotted line on the monochromatic images.

Figure 12 show the row profiles for the two regions from the full spectrum (blue line) and scatter free monochromatic spectrum (red line). Table 8 shows the contrast values calculated from the regions indicated in figure 12. From this data it can be seen that although there is clearly a higher standard deviation in the points of the monochromatic images the contrast is higher than for the full spectrum images with an increase of about $45 \%$. Table 9 shows the CNR values for the full spectrum and scatter free data. These were calculated as an additional measure of the image quality. It was found that there 
was around an $18 \%$ increase in the CNR for the scatter free image. This further proves the validity of this method for producing scatter free images for low contrast imaging in mammography.

Region 1

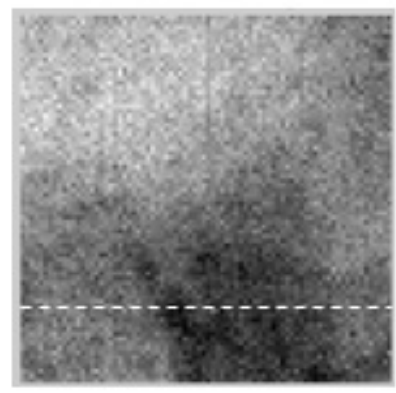

Region 2

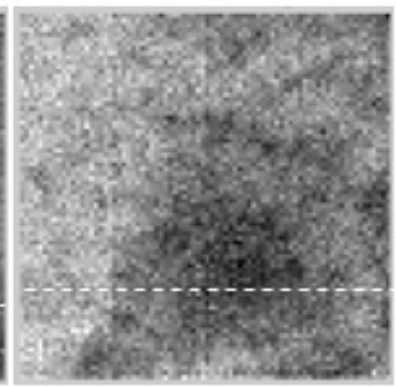

Figure 11. images of region 1 and region 2 of the Rachel phantom using a full $45 \mathrm{kVp}$ energy spectrum. The images line up with the top three fifths of the monochromatic images shown in figure 8 and the dotted lines show where the row profile was taken which is the same row taken from the monochromatic images.

Table 8. contrast values for the single row profiles for the full spectrum and monochromatic scatter free data.

\begin{tabular}{lll}
\hline & Full energy contrast & Scatter-free contrast \\
\hline Region 1 & $0.053 \pm 0.002$ & $0.11 \pm 0.01$ \\
Region 2 & $0.054 \pm 0.003$ & $0.10 \pm 0.01$ \\
\hline
\end{tabular}

Table 9. CNR values for the single row profiles of the full spectrum and monochromatic scatter free data.

\begin{tabular}{lll}
\hline & Full energy CNR & Scatter-free CNR \\
\hline Region 1 & $289 \pm 7$ & $364 \pm 8$ \\
Region 2 & $283 \pm 6$ & $339 \pm 7$ \\
\hline
\end{tabular}

\section{Conclusions}

Mammography requires a high image quality as the details being detected are low in contrast. Current systems use an anti-scatter grid to remove scattered X-rays but this method also removes a proportion of primary beam therefore an alternative method of scatter removal was investigated.

A monochromatic system using a conventional X-ray source combined with a pixellated spectroscopic detector was developed. By imaging using a monochromatic beam and 


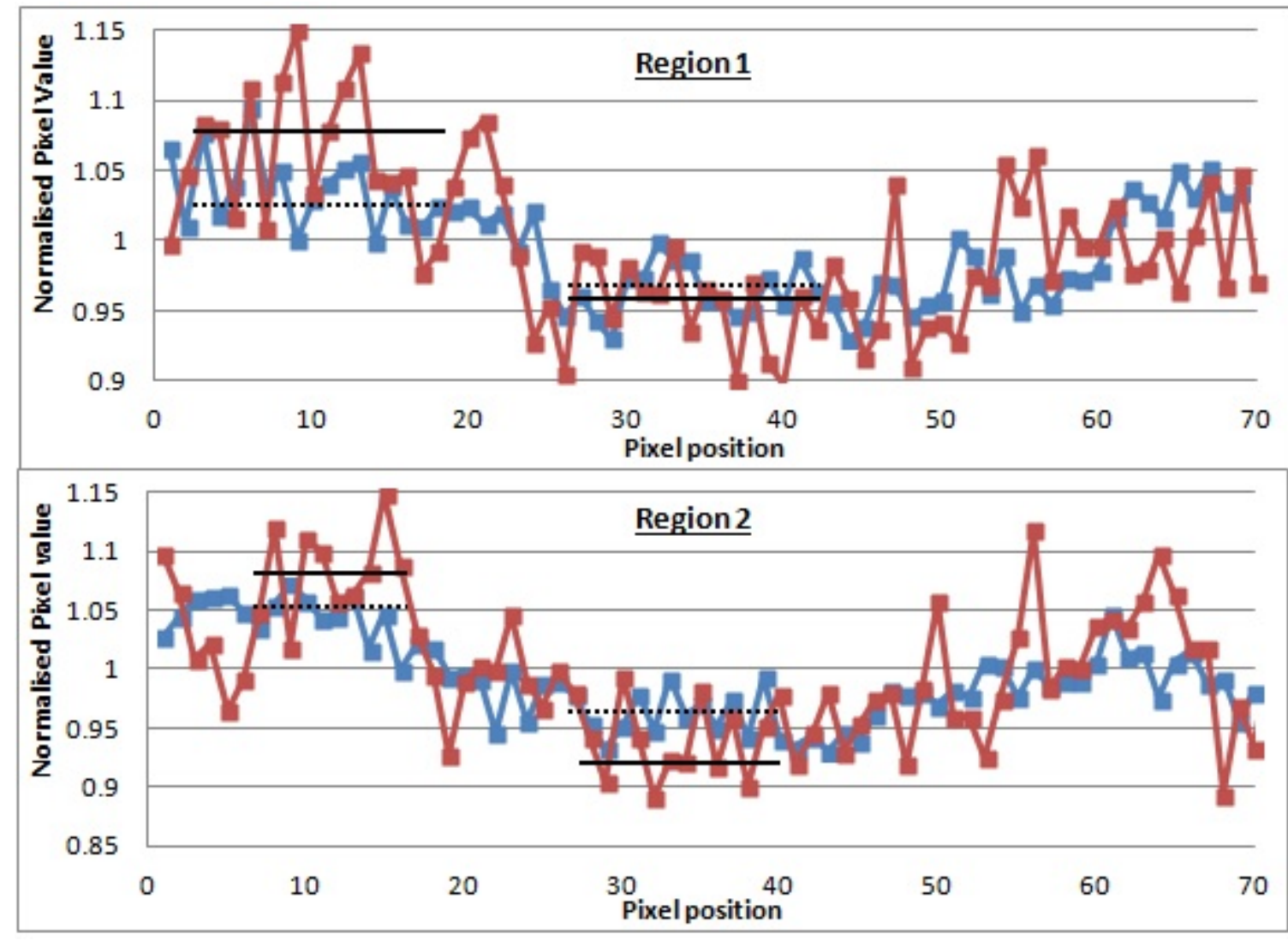

Figure 12. plots of a single row from region 1 (top) and a single row from region 2 (bottom) comparing the full energy spectrum images (blue line) and scatter-free images (red lines), contrast values are estimated using the points indicated where the dotted line represents points from the scatter-free data and the solid line represents points from the full spectrum data. The pixel values are normalised to the mean pixel value.

then windowing pixel by pixel the detected spectrum around the monochromatic peak, additional Compton scattered photons were removed from the image producing a scatter free image. The use of a conventional X-ray source over a synchrotron source allows for the production of a monochromatic beam without the size and expense of a synchrotron. The addition of the spectroscopic detector means that scatter removal not previously possible, as a result of little information about the energy of the photons, is now achievable.

Images of a low contrast test object and a breast phantom were acquired and comparisons of the image quality before and after windowing of the energy spectrum were made. Results of contrast improvement have been presented for the method of producing scatter free images for the use of mammography. Promising improvements of around $50 \%$ in contrast have been seen when imaging low contrast details. This was confirmed from images of an anthropomorphic breast phantom.

The CNR was also calculated for both the low contrast test object and the breast 
phantom. It was found that when comparing CNR values calculated from images acquired with a similar dose that there was an increase in the CNR for the scatter free images. Although the CNR for the polychromatic spectrum images is greater due to the much higher statistics they feature, the IQF, which removes the dependency upon dose is greater for the monochromatic scatter-free data, demonstrating the effectiveness of the technique in improving the image quality for a given dose. The low intensity of the monochromatic data is due to the efficiency of our setup as a large amount of the beam was absorbed in the diffraction process and the maximum tube current itself was very low. It has, however, been shown in work by other groups that an imaging efficiency in line with current radiographic methods is possible with a more complex setup and accurate allignment [Baldelli et al., 2005] [Gambaccini et al., 1999].

Future work on this area of research is to develop a system capable of imaging in a clinical environment. The current detector area is too small for clinical imaging therefore a larger detector area is needed. A $10 \mathrm{~cm}$ x $10 \mathrm{~cm}$ HEXITEC prototype has been developed which demonstrates the capability of producing larger area detectors [Wilson et al., 2015]. Also as HEXITEC is designed for low rates and medical imaging requires high rates, a detector that compromises between the energy resolution and the rate is needed. To carry out this method an energy resolution equal to the FWHM of the monochromatic peak is needed to distinguish it from the scatter so a lesser resolution could be used, still producing the same result.

The spatial resolution of the imaging system used is currently not suitable for clinical imaging due to the size of details such as calcifications being smaller than the pixel pitch of the detector, $250 \mu \mathrm{m}$. There are developments into sub pixel resolution algorithms for HEXITEC that uses the centroid of the charge distribution from neighbouring pixels to deduce the location of an interaction in the sub pixel scale, this will be the subject of further investigation.

Finally, an X-ray tube with a current that matches those used in mammography is required. The tube used in this study has a maximum tube current of $100 \mu \mathrm{A}$, which is around 100 times smaller the current used in conventional mammography (in the $10 \mathrm{~mA}$ range) [Bushberg, 2002]. This increase in tube current would improve the detection time from around 40 minutes to seconds. This time would be compatible with clinical requirements.

\section{Acknowledgments}

This work was partially funded by the Engineering and Physical Sciences Research Council, EPSRC (Grant number: EP/H046577/1).

\section{References}

[Ahmed, 2014] Ahmed, S. (2014). Physics and Engineering of Radiation Detection. Elsevier Science. [Aichinger et al., 2011] Aichinger, H., Dierker, J., Joite-Barfuß, S., and Säbel, M. (2011). Radiation 
exposure and image quality in X-ray diagnostic radiology: physical principles and clinical applications. Springer Science \& Business Media.

[Baldelli et al., 2003] Baldelli, P., Taibi, A., Tuffanelli, A., and Gambaccini, M. (2003). Quasimonochromatic x-rays for diagnostic radiology. Physics in medicine and biology, 48(22):3653.

[Baldelli et al., 2005] Baldelli, P., Taibi, A., Tuffanelli, A., Gilardoni, M., and Gambaccini, M. (2005). A prototype of a quasi-monochromatic system for mammography applications. Physics in medicine and biology, 50(10):2225.

[Ballabriga et al., 2011] Ballabriga, R., Blaj, G., Campbell, M., Fiederle, M., Greiffenberg, D., Heijne, E., Llopart, X., Plackett, R., Procz, S., Tlustos, L., et al. (2011). Characterization of the medipix3 pixel readout chip. Journal of Instrumentation, 6(01):C01052.

[Besson et al., 2002] Besson, G. M., Koch, A., Tesic, M., Sottoriva, R., Prieur-Drevron, P., Munier, B., Calais, E., and DeGroot, P. (2002). Design and evaluation of a slot-scanning full-field digital mammography system. In Medical Imaging 2002, pages 457-468. International Society for Optics and Photonics.

[Boone et al., 2001] Boone, J. M., Nelson, T. R., Lindfors, K. K., and Seibert, J. A. (2001). Dedicated breast ct: Radiation dose and image quality evaluation 1. Radiology, 221(3):657-667.

[Brahme, 2014] Brahme, A. (2014). Comprehensive Biomedical Physics. Elsevier Science.

[Burattini et al., 1995] Burattini, E., Cossu, E., Di Maggio, C., Gambaccini, M., Indovina, P., Marziani, M., Pocek, M., Simeoni, S., and Simonetti, G. (1995). Mammography with synchrotron radiation. Radiology, 195(1):239-244.

[Bushberg, 2002] Bushberg, J. (2002). The Essential Physics of Medical Imaging. Lippincott Williams \& Wilkins.

[Bushberg et al., 2011] Bushberg, J., Seibert, J., Leidholdt, E., and Boone, J. (2011). The Essential Physics of Medical Imaging. Wolters Kluwer Health.

[Castelli et al., 2011] Castelli, E., Tonutti, M., Arfelli, F., Longo, R., Quaia, E., Rigon, L., Sanabor, D., Zanconati, F., Dreossi, D., Abrami, A., et al. (2011). Mammography with synchrotron radiation: first clinical experience with phase-detection technique. Radiology, 259(3):684-694.

[Dance et al., 2000] Dance, D., Skinner, C., Young, K., Beckett, J., and Kotre, C. (2000). Additional factors for the estimation of mean glandular breast dose using the uk mammography dosimetry protocol. Physics in medicine and biology, 45(11):3225.

[Darboux and Dinten, 1997] Darboux, M. and Dinten, J.-M. (1997). Physical-model-based scatter correction in mammography. In Medical Imaging 1997, pages 405-410. International Society for Optics and Photonics.

[Ducote and Molloi, 2010] Ducote, J. and Molloi, S. (2010). Scatter correction in digital mammography based on image deconvolution. Physics in medicine and biology, 55(5):1295.

[Evans, 2007] Evans, P. (2007). Calculation of x-ray spectra emerging from an x-ray tube. part i. electron penetration characteristics in x-ray targets. Medical Physics, 34(6):2164-2174.

[Flower, 2012] Flower, M. (2012). Webb's Physics of Medical Imaging, Second Edition. Series in Medical Physics and Biomedical Engineering. Taylor \& Francis.

[Freund et al., 1996] Freund, A. K., Munkholm, A., and Brennan, S. (1996). X-ray diffraction properties of highly oriented pyrolytic graphite. In SPIE's 1996 International Symposium on Optical Science, Engineering, and Instrumentation, pages 68-79. International Society for Optics and Photonics.

[Gambaccini et al., 1999] Gambaccini, M., Tuffanelli, A., Taibi, A., Fantini, A., and Del Guerra, A. (1999). Bragg-diffraction-based quasi-monochromatic source for mammography using mosaic crystals. In SPIE's International Symposium on Optical Science, Engineering, and Instrumentation, pages 174-184. International Society for Optics and Photonics.

[Green et al., 2014] Green, F., Veale, M., Wilson, M., Seller, P., Scuffham, J., and Pani, S. (2014). A novel approach to scatter-free imaging for the improvement of breast cancer detection. Journal of Instrumentation, 9(12):C12013.

[Green et al., 2015] Green, F., Veale, M., Wilson, M., Seller, P., Scuffham, J., and Pani, S. (2015). 
Scatter-free breast imaging using a monochromator coupled to a pixellated spectroscopic detector. In SPIE Medical Imaging, pages 94125G-94125G. International Society for Optics and Photonics.

[Longo et al., 2014] Longo, R., Tonutti, M., Rigon, L., Arfelli, F., Dreossi, D., Quai, E., Zanconati, F., Castelli, E., Tromba, G., and Cova, M. A. (2014). Clinical study in phase-contrast mammography: image-quality analysis. Philosophical Transactions of the Royal Society of London A: Mathematical, Physical and Engineering Sciences, 372(2010):20130025.

[Lundqvist et al., 2003] Lundqvist, M., Bergström, D., Cederström, B., Chmill, V., Chuntonov, A., Danielsson, M., and Åslund, M. (2003). Physical evaluation of a prototype for the sectra microdose mammography system. In Digital Mammography, pages 12-16. Springer.

[Melzer et al., 2008] Melzer, T., Cook, N., Butler, A., Watts, R., Anderson, N., Tipples, R., and Butler, P. (2008). Spectroscopic biomedical imaging with the medipix2 detector. Australasian Physics $\&$ Engineering Sciences in Medicine, 31(4):300-306.

[Moeckli et al., 2000] Moeckli, R., Verdun, F., Fiedler, S., Pachoud, M., Schnyder, P., and Valley, J. (2000). Objective comparison of image quality and dose between conventional and synchrotron radiation mammography. Physics in medicine and biology, 45(12):3509.

[Monnin et al., 2007] Monnin, P., Gutierrez, D., Bulling, S., Guntern, D., and Verdun, F. (2007). A comparison of the performance of digital mammography systems. Medical physics, 34(3):906-914.

[Oppelt, 2011] Oppelt, A. (2011). Imaging Systems for Medical Diagnostics: Fundamentals, Technical Solutions and Applications for Systems Applying Ionizing Radiation, Nuclear Magnetic Resonance and Ultrasound. Wiley.

[Optigraph, 2011] Optigraph (2011). http://www.optigraph.eu/.

[Pinsky et al., 2014] Pinsky, L., Idarraga-Munoz, J., Kroupa, M., Son, H., Stoffle, N., Semones, E., Bahadori, A., Turecek, D., Pospíšil, S., Jakubek, J., et al. (2014). Medipix in space on-board the iss. Journal of radiation research, 55(suppl 1):i62-i63.

[Poludniowski et al., 2009] Poludniowski, G., Landry, G., DeBlois, F., Evans, P., and Verhaegen, F. (2009). Spekcalc: a program to calculate photon spectra from tungsten anode x-ray tubes. Physics in medicine and biology, 54(19):N433.

[Poludniowski, 2007] Poludniowski, G. G. (2007). Calculation of x-ray spectra emerging from an x-ray tube. part ii. x-ray production and filtration in x-ray targets. Medical physics, 34(6):2175-2186.

[Ronckers et al., 2005] Ronckers, C. M., Erdmann, C. A., and Land, C. E. (2005). Radiation and breast cancer: a review of current evidence. Breast Cancer Res, 7(1):21-32.

[Shikhaliev and Fritz, 2011] Shikhaliev, P. M. and Fritz, S. G. (2011). Photon counting spectral ct versus conventional ct: comparative evaluation for breast imaging application. Physics in medicine and biology, 56(7):1905.

[Sprawls, 1995] Sprawls, P. (1995). Physical Principles of Medical Imaging. Medical Physics Pub.

[Thunberg et al., 2002] Thunberg, S. J., Francke, T., Egerstrom, J., Eklund, M., Ericsson, L., Kristoffersson, T., Peskov, V. N., Rantanen, J., Sokolov, S., Svedenhag, P., et al. (2002). Evaluation of a photon-counting mammography system. In Medical Imaging 2002, pages 202208. International Society for Optics and Photonics.

[Veale et al., 2012] Veale, M., Bell, S., Seller, P., Wilson, M., and Kachkanov, V. (2012). X-ray microbeam characterization of a small pixel spectroscopic cdte detector. Journal of Instrumentation, $7(07): \mathrm{P} 07017$.

[Wang et al., 2015] Wang, A., Shapiro, E., Yoon, S., Ganguly, A., Proano, C., Colbeth, R., Lehto, E., and Star-Lack, J. (2015). Asymmetric scatter kernels for software-based scatter correction of gridless mammography. In SPIE Medical Imaging, pages 94121I-94121I. International Society for Optics and Photonics.

[Wilson et al., 2015] Wilson, M., Dummott, L., Duarte, D., Green, F., Pani, S., Schneider, A., Scuffham, J., Seller, P., and Veale, M. (2015). A $10 \mathrm{~cm} \times 10 \mathrm{~cm}$ cdte spectroscopic imaging detector based on the hexitec asic. Journal of Instrumentation, 10(10):P10011.

[Wilson et al., 2013] Wilson, M. D., Bell, S. J., Cernik, R. J., Christodoulou, C., Egan, C. K., O'Flynn, D., Jacques, S., Pani, S., Scuffham, J., Seller, P., et al. (2013). Multiple module pixellated cdte 
spectroscopic x-ray detector. Nuclear Science, IEEE Transactions on, 60(2):1197-1200. 